\title{
Troubleshooting Liquid Chromatographic Instrumentation and Methods
}

\author{
Lane C. Sander \\ National Institute of Standards and Technology, \\ Gaithersburg, MD 20899, USA \\ lane.sander@nist.gov
}

Video DOI: http://doi.org/10.18434/T4DK5T

Key words: instrumentation; method development; problem solving; system performance; troubleshooting.

Accepted: December 1, 2016

Published: January 12, 2017

https://doi.org/10.6028/jres.122.003

\section{Summary}

The ability to assess analytical performance is a fundamental component of measurement science. Ultimately, measurement quality depends on the proper function of instrumentation in combination with appropriate methods. Evidence of proper function is typically provided by the instrumentation, chromatograms, and the resulting data generated. Chromatographic peak shape is diagnostic of problems that originate with the instrumentation as well as the method and sample. Once a problem has been clearly identified, steps can be taken to resolve the issue. This presentation will describe common problems than may be encountered, with representative illustrations of the resulting effects on chromatographic performance. $^{1}$

\footnotetext{
${ }^{1}$ Contribution of the National Institute of Standards and Technology. Not subject to copyright. Certain commercial equipment, instruments, or materials are identified to specify adequately the experimental procedure. Such identification does not imply recommendation or endorsement by the National Institute of Standards and Technology, nor does it imply that the materials or equipment identified are the best available for the purpose.
} 